\title{
Caso clínico: manejo anestésico en corrección de atresia esofágica en recién nacido
}

\section{Clinical report: anesthetic management in correction of esophageal atresia in a newborn}

Daniel Rivera MD. ${ }^{1}$, Juan Parra MD. ${ }^{1}$, Danny Rodríguez MD. ${ }^{1}$

\begin{abstract}
Esophageal atresia (AE), with or without tracheoesophageal fistula (FTE), is a pathology congenital that has a frequency of $1: 2,500-4,500$ live births. It has been reported that Up to $11 \%$ of all cases are voluntarily terminated in the prenatal period, a $2 \%$ die in utero and $87 \%$ end up in viable live births. There is a predominance for male sex, week 38 being the most frequent gestational age of documentation. The purpose of the clinical case described below is to highlight the plan A successful anesthetic that was successfully in a fourth level university hospital, in a full-term newborn with AE and FTE, taking into account the pre, intra and postoperative recommendations.
\end{abstract}

\section{RESUMEN}

La atresia esofágica (AE), con o sin fístula traqueoesofágica (FTE), es una patología congénita que tiene una frecuencia de 1: 2.500 - 4.500 nacimientos vivos. Se ha informado que hasta el $11 \%$ de todos los casos se terminan voluntariamente en el período prenatal, un $2 \%$ muere en el útero y el $87 \%$ termina en nacimientos vivos viables. Hay un predominio del sexo masculino, siendo la semana 38 la edad gestacional de documentación más frecuente. El propósito del caso clínico que se describe a continuación es resaltar el plan. Un anestésico exitoso que tuvo éxito en un hospital universitario de cuarto nivel, en un recién nacido a término con EA y FTE, teniendo en cuenta Recomendaciones pre, intra y postoperatorias.

\section{Key words:}

Esophageal atresia, newborn,

anasthetic managment

\section{Palabras clave:}

Atresia esofágica, recién nacido, manejo anestésico

Hospital Universitario Hernando Moncaleano Perdomo, Colombia.

Fecha de ingreso: 19 de agosto de 2019

Fecha de aceptación: 22 de septiembre de 2019

\section{ORCID}

https://orcid.org/0000-0002-5221-1024

Correspondencia:

Email: juansparra@gmail.com 


\section{Introducción}

a atresia esofágica (AE), con o sin fístula traqueoesofágica (FTE), es una patología congénita que tiene una frecuencia de 1:2.500 - 4.500 nacidos vivos[1]. Se ha reportado que hasta un $11 \%$ de todos los casos son terminados voluntariamente en el período prenatal, un $2 \%$ fallecen in útero y $87 \%$ terminan en nacidos vivos viables. Existe un predominio por el sexo masculino, siendo la semana 38 la edad gestacional de documentación más frecuente[2].

La forma más frecuente por clasificación de Gross, es el tipo $C$ en un $86 \%$ de los casos (AE + FTE distal). Siendo relevante que hasta un $60 \%$ de los casos tienen asociado una anomalía congénita. Aproximadamente el $50 \%$ de los pacientes puede tener asociado VACTERL (anomalías vertebrales, atresia anal, fistula traqueoesofágica, anomalías radiales, cardiovasculares, renales o de extremidades), siendo imperativo los estudios adicionales ya que pueden modificar tanto el plan anestésico como el quirúrgico en estos pacientes.

El propósito del caso clínico que a continuación se expone, es poner de manifiesto el plan anestésico exitoso que se desarrolló en un hospital universitario de cuarto nivel de atención, en un recién nacido a término con AE y FTE, teniendo en consideración las recomendaciones pre, intra y postoperatorias.

\section{Caso clínico}

Paciente de sexo masculino, sin antecedentes prenatales de importancia, fruto de gestación de 37,3 semanas de madre de 32 años de edad, nacido por cesárea por polihidramnios. Adaptación neonatal espontánea, APGAR 8-9-10/10, quien durante prueba de permeabilidad esofágica se evidencia imposibilidad de paso de sonda orogástrica considerando alta probabilidad de atresia esofágica.

Se traslada a la Unidad de Cuidados Intensivos neonatal para monitoria continua, donde se inicia nutrición parenteral, manejo de secreciones orales con sonda de doble lumen con irrigación y aspiración continua y manejo multidisciplinario. La radiografía de tórax evidenció presencia de la sonda orogástrica a nivel de T2-T3, además de aire en la cámara gástrica, por lo cual y por concepto de neumología se consideró presencia fístula traqueoesofágica. Se confirma diagnóstico de atresia esofágica por cirugía pediátrica indicándose necesidad de corrección de defecto congénito por toracotomía con aislamiento pulmonar derecho. El hemograma, la función renal, los electro- litos y el ecocardiograma transtorácico no sugirieron patologías congénitas adicionales asociadas.

Es valorado por anestesiología quien considera puede ser llevado a corrección de atresia esofágica y fístula traqueoesofágica por toracotomía (riesgo quirúrgico alto), haciéndose énfasis en la necesidad de realizar preoperatoriamente fibrobroncoscopia por el Servicio de Neumología, para definir abordaje definitivo de la vía aérea según nivel de la FTE.

Se traslada neonato a salas de cirugía, confirmándose acceso periférico con yelco \#24 en miembro superior izquierdo funcional. Previa monitoria básica (presión arterial no invasiva, saturación de oxígeno, electrocardiograma y temperatura) se hace inducción inhalatoria con sevorane. Bajo plano anestésico adecuado y ventilación espontánea (apoyando con máscara facial), se avanza fibrobroncoscopio (por adaptador de mouth) evidenciándose presencia de fístula traqueoesofágica carinal posterior (Figura 1). Se confirma ingreso a cavidad gástrica al avanzar fibrobroncoscopio por luz de fístula.

Confirmadas luces bronquiales, se avanza catéter fogarty \#3 a través de las cuerdas vocales por laringoscopia directa con hoja recta \#00 (Cormack II). Se confirma adecuado aislamiento pulmonar por fibrobronscopia, se insufla manguito del catéter fogarty en total con $2,5 \mathrm{~cm}$ en bronquio fuente derecho. Previa administración de bolo de propofol (3 $\mathrm{mg} / \mathrm{kg}$ ), remifentanilo $(2 \mathrm{mcg} / \mathrm{kg})$ y rocuronio $(0,6 \mathrm{mg} / \mathrm{kg})$, se realiza nueva laringoscopia, avanzando tubo orotraqueal

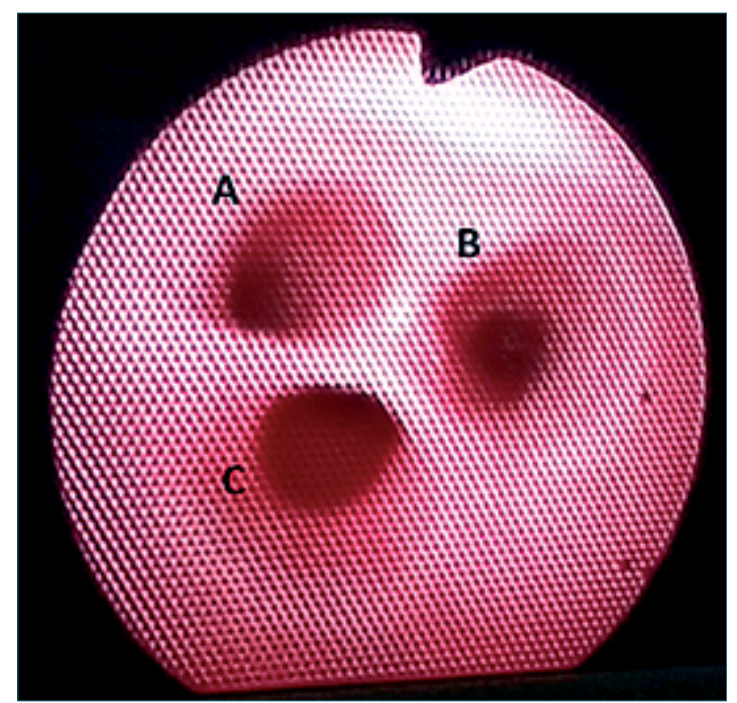

Figura 1. Visualización fibrobroncoscópica de bronquio fuente izquierdo (A), bronquio fuente derecho (B) y luz de fístula traqueoesofágica carinal posterior (C). 
\#3.0 con balón, fijándolo a 8 cm previa confirmación de capnografía y auscultación simétrica.

Se posiciona a paciente en decúbito lateral izquierdo junto con el equipo quirúrgico haciendo protección de los puntos de presión y de las zonas declive. Previa asepsia y antisepsia se realiza bloqueo ecoguiado del erector de la espina a nivel de T5 con aguja de bloqueo de $50 \mathrm{~mm}$, administrándose en total volumen de $3 \mathrm{ml}(1 \mathrm{ml}$ de bupivacaina al 0,5\% $+1 \mathrm{ml}$ de lidocaína al $1 \%$ con epinefrina $+1 \mathrm{ml}$ de solución salina al 0,9\%) sin complicaciones.

Se da mantenimiento anestésico balanceado con remifentanilo en promedio a $0,2 \mathrm{mcg} / \mathrm{kg} / \mathrm{min}$ y sevorane manteniendo $M A C>0,6$. Se mantuvo ventilación protectiva durante aislamiento pulmonar (VC 4-6 ml/kg, FR > $25 \mathrm{rpm}$ e hipercapnia permisiva) sin documentarse problemas ventilatorios durante el procedimiento. Se dio coadyuvancia analgésica con ketamina $(0,25 \mathrm{mg} / \mathrm{kg})$, dipirona $(50 \mathrm{mg} / \mathrm{kg})$ y dexametasona $(0,15 \mathrm{mg} / \mathrm{kg})$. Se expresó por parte del equipo quirúrgico aislamiento pulmonar efectivo con campo quirúrgico adecuado. El tiempo quirúrgico fue de aproximadamente 2 horas y 30 minutos.

Finalizado el procedimiento se hace reversión de la relajación neuromuscular con sugammadex a dosis de $2 \mathrm{mg} / \mathrm{kg}$. Se retira catéter fogarty y se extuba al paciente posterior a verificar mecánica ventilatoria y nivel de alertamiento adecuados. Se traslada a la Unidad de Cuidados Intensivos con cánula nasal, sin sig- nos clínicos que sugirieran dolor. El paciente es dado de alta a las 48 horas del procedimiento quirúrgico.

\section{Discusión}

Es fundamental tener en consideración que el abordaje de los pacientes con AE confirmada requiere de un abordaje multimodal en el pre, intra y postoperatorio (Tabla 1).

Dentro de las recomendaciones preoperatorias, una vez se tenga la sospecha de una AE (recién nacidos con polihidramnios, no posibilidad de paso de sonda orogástrica, ausencia de burbuja gástrica en ecografía prenatal)[3], el paciente deberá ser trasladado a la brevedad a la Unidad de Cuidados Intensivos. La prioridad será asegurar un acceso venoso para iniciar líquidos parenterales de mantenimiento, asegurar euvolemia y normoglicemia. Se deberá hacer manejo adecuado de las secreciones orales ya que se puede desarrollar rápidamente cuadros de atragantamiento, distrés respiratorio y broncoaspiración. En lo posible se debe mantener ventilación espontánea, dado el riesgo de insuflación gástrica con sistemas de asistencias ventilatoria no invasiva con CPAP. Se puede posicionar al paciente en decúbito prono con trendelemburg para mitigar el riesgo de broncoaspiración[1].

Entre los estudios complementarios está la rea-

\section{Tabla 1. Consideraciones anestésicas para el manejo pre, intra y postoperatorio}

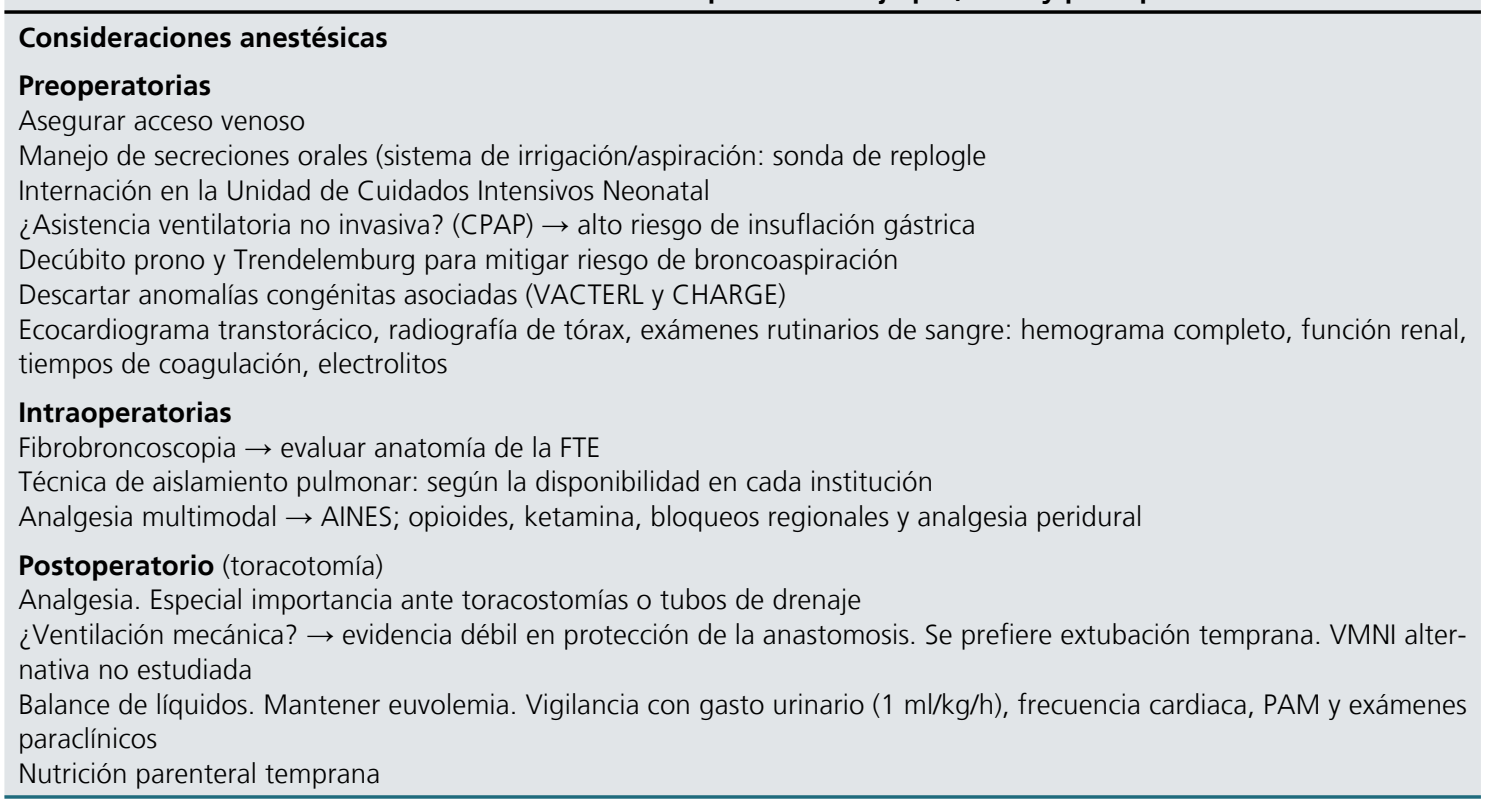


lización de un ecocardiograma transtorácico, con el cual se descartará la presencia de cardiopatías congénitas o de un arco aórtico derecho que definirá el abordaje quirúrgico. La radiografía de tórax expondrá la presencia o no de burbuja gástrica lo cual confirmaría la presencia de FTE, lo cual es fundamental en el abordaje de la vía aérea. Los exámenes rutinarios de sangre, encaminados a descartar patologías congénitas asociadas, incluyen un hemograma completo, tiempos de coagulación, química sanguínea completa y grupo sanguíneo previendo que el procedimiento tiene alto riesgo de sangrado[1].

Dentro de las recomendaciones intraoperatorias, la prioridad es la realización de una fibrobroncoscopia dado que la presencia de una FTE pericanial alertara al anestesiólogo de posibles dificultades ventilatorias durante el procedimiento[5]. Igualmente, la asistencia con fibrobroncoscopia para la ubicación de los dispositivos para el aislamiento pulmonar gana especial importancia[6].

En cuanto a las estrategias analgésicas el manejo deberá ir encaminado a una estrategia multimodal: AINEs, opioides, ketamina, bloqueos regionales y analgesia peridural. La estrategia analgesia deberá estar encaminada a la experiencia personal. En nuestro caso se optó por un bloqueo erector de la espina, teniendo en consideración que cada vez se tienen más reportes exitosos con esta técnica analgésica en neonatos llevados a toracotomía[7].

Existen ciertas recomendaciones para el manejo postoperatorio de los pacientes llevados a correcciones por toracotomía, sin embargo, se salen del propósito del presente reporte (Tabla 1).

\section{Conclusiones}

Presentamos el caso de un neonato llevado a corrección de una $\mathrm{AE}+\mathrm{FTE}$, teniendo en consideración el plan anestésico para el manejo de la vía aérea, aislamiento pulmonar y el plan analgésico postoperatorio. La frecuencia de la AE no es despreciable y se puede presentar en el ejercicio profesional de cualquier anestesiólogo general que trabaje en un hospital de cuarto nivel de atención como el nuestro. De ahí la importancia de conocer las recomendaciones y la evidencia disponible, aunque escasa, del abordaje de estos pacientes.

\section{Referencias}

1. Rod WH, Elizabeth JP, King S. Peri-operative management of neonates with oesophageal atresia and tracheo-oesophageal fistula. Paediatric Respiratory Reviews 2016; 19:3-9. https://doi.org/10.1016/j. prrv.2016.01.002

2. Garne E, Loane M, Dolk H. a EUROCAT Working Group. Gastrointestinal malformations: impact of prenatal diagnosis on gestational age at birth. Paediatric and Perinatal Epidemiology 2007;21:370-5. https://doi.org/10.1111/j.13653016.2007.00826.x

3. Sfeir R, Bonnard A, Khen-Dunlop $N$, Auber F, Gelas T, Michaud L, et al. Esophageal atresia: data from a national cohort. J Pediatr Surg. 2013 Aug;48(8):1664-9. https://doi.org/10.1016/j. jpedsurg.2013.03.075 PMID:23932604

4. Nasr A, McNamara PJ, Mertens L, Levin D, James A, Holtby $H$, et al. Is routine preoperative 2 -dimensional echocardiography necessary for infants with esophageal atresia, omphalocele, or anorectal malformations? J Pediatr Surg. 2010 May;45(5):8769. https://doi.org/10.1016/j. jpedsurg.2010.02.002 PMID:20438917

5. Andropoulos DB, Rowe RW, Betts JM. Anaesthetic and surgical airway management during tracheo-oesophageal fistula repair. Paediatr Anaesth. 1998;8(4):313-9. https://doi.org/10.1046/

j.1460-9592.1998.00734.x PMID:9672929

6. Letal M, Theam M. Paediatric lung isolation. BJA Educ. 2017;17(2):57-62. https://doi. org/10.1093/bjaed/mkw047.

7. Adam C. Adler, Michael M. Yim, Arvind Chandrakantan. Erector spinae plane catheter for neonatal thoracotomy: a potentially safer alternative to a thoracic epidural. Can J Anesth. 2019. https://10.1007/s12630-01901296-w

8. Muñoz F, Cubillos J, Bonilla AJ. Erector spinae plane block for postoperative analgesia in pediatric oncological thoracic surgery. Can J Anesth. 2019. https://10.1007/s12630-0170894-0 